\title{
Current Reference Circuit for Subthreshold CMOS LSIs
}

\author{
Ken Ueno, Tetsuya Asai, and Yoshihito Amemiya \\ Department of Electrical Engineering, Hokkaido University, 060-0814, Japan \\ E-mail: k_ueno@sapiens-ei.eng.hokudai.ac.jp
}

\section{INTRODUCTION}

Intelligent network systems for the future will require a great number of smart sensor LSIs that measure various physical data in surroundings. These LSIs have to operate with a low power, tens of microwatts or less, because they will probably be placed in non-ideal environments where energy for operation cannot be obtained sufficiently. To achieve such ultra-low power operation, CMOS circuits in sensor LSIs have to be operated in the subthreshold region of MOSFETs. For this purpose, we need to develop a circuit that provides a small reference current of less than tens of nanoamperes for the subthreshold CMOS circuits. We propose such a reference current circuit that is insensitive to temperature and power supply voltage.

Current references for subthreshold CMOS circuits have been proposed by Oguey and Aebischer [1], and Hirose and others $[2,3]$. The former current reference, however, shows a positive temperature coefficient of output current. The latter shows a zero temperature coefficient but consists of a complex circuit with many MOSFETs. Therefore, they are unsuitable for our purpose. The current reference circuit we propose will solve these problems and can be used for lowpower subthreshold MOS LSIs. The following describes the details of our current reference circuit.

\section{Circuit CONFIGURATION}

\section{A. Operation Principle}

Figure 1 shows the current reference circuit we propose. The circuit consists of a bias-voltage subcircuit and a current-source subcircuit. Bias voltage $V_{B}$ for MOS resistor $\mathrm{M}_{3}$ is generated by a diode-connected transistor $\mathrm{M}_{4}$. The current-source subcircuit accepts bias voltage $V_{B}$ and generates reference current $I_{O U T}$. All MOSFETs are operated in the subthreshold region except for transistors $\mathrm{M}_{3}$ and $\mathrm{M}_{4}$.

The current $I_{D}$ in a subthreshold-operated MOSFET can be given by

$$
I_{D}=K I_{0} \exp \left(\frac{V_{G S}-V_{T H}}{\eta V_{T}}\right),
$$

where $K$ is the aspect ratio $(=\mathrm{W} / \mathrm{L})$ of the MOSFET, $I_{0}$ $\left(=\mu C_{O X}(\eta-1) V_{T}^{2}\right)$ is a process-dependent parameter, $\mu$ is the mobility, $C_{O X}$ is the gate-oxide capacitance, $\eta$ is the subthreshold slope factor, $V_{T H}$ is the threshold voltage, $V_{T}$ $\left(=k_{B} T / q\right)$ is the thermal voltage, $k_{B}$ is the Boltzmann constant, $T$ is the absolute temperature, and $q$ is the elementary charge [4].

Current $I_{\text {BIAS }}$ flowing in each path of the bias-voltage subcircuit is determined by the ratio of the aspect ratios of $M_{1}$ and $M_{2}$ and the resistance of MOS resistor $M_{3}$. The current is given by

$$
I_{B I A S}=K_{3} \mu C_{O X}\left(V_{B}-V_{T H}\right) \eta V_{T} \ln \left(K_{2} / K_{1}\right) .
$$

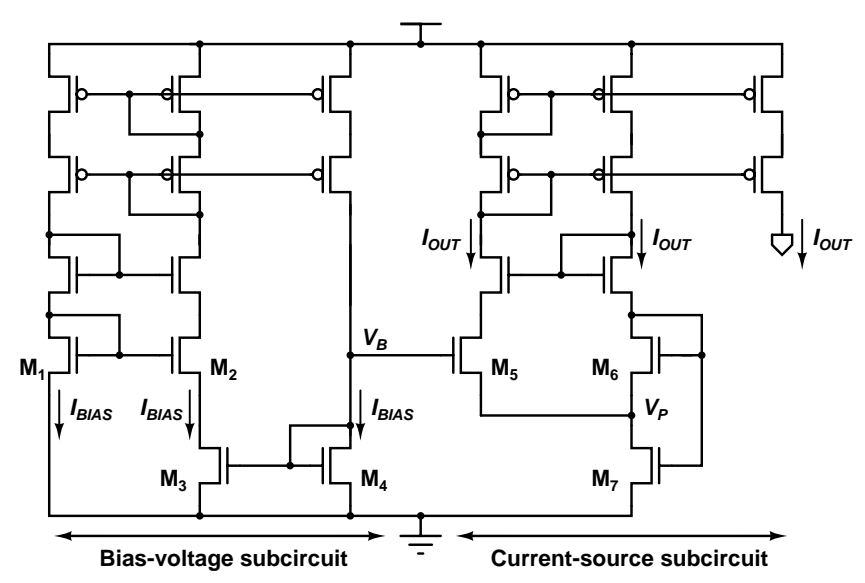

Fig. 1. Schematic of the proposed current reference circuit.

The diode-connected transistor $\mathrm{M}_{4}$ is operated in the strong inversion and saturation region. Its drain current $I_{B I A S}$ is given by

$$
I_{B I A S}=\frac{K_{4} \mu C_{O X}}{2}\left(V_{B}-V_{T H}\right)^{2} .
$$

Because the currents in transistors $\mathrm{M}_{3}$ and $\mathrm{M}_{4}$ are the same, Eqs. (2) and (3) show that $V_{B}$ is

$$
V_{B}=V_{T H}+\frac{2 K_{3}}{K_{4}} \eta V_{T} \ln \left(\frac{K_{2}}{K_{1}}\right)
$$

Output current $I_{\text {OUT }}$ through transistor $\mathrm{M}_{5}$ operating in the subthreshold region can be given by

$$
I_{\text {OUT }}=K_{5} I_{0} \exp \left(\frac{V_{B}-V_{P}-V_{T H 5}}{\eta V_{T}}\right) .
$$

The source voltage $V_{P}$ of transistor $\mathrm{M}_{5}$ can be given by

$$
V_{P}=V_{G S 7}-V_{G S 6}=\eta V_{T} \ln \left(\frac{2 K_{6}}{K_{7}}\right)-\Delta V_{T H 76},
$$

where $\Delta V_{T H 76}$ is the difference between the threshold voltages of $\mathrm{M}_{6}$ and $\mathrm{M}_{7}$ (including the body effect in the transistors). From Eqs. (4), (5), and (6), we find that

$$
I_{\text {OUT }}=K_{5} I_{0} \exp \left(\frac{\Delta V_{T H}}{\eta V_{T}}\right) \cdot \frac{K_{7}}{2 K_{6}}\left(\frac{K_{2}}{K_{1}}\right)^{2 K_{3} / K_{4}},
$$

where $\Delta V_{T H}\left(=\Delta V_{T H 76}-\Delta V_{T H 54}\right)$ is the difference between the threshold voltages of transistors $\mathrm{M}_{4}-\mathrm{M}_{7}$. This way, we can obtain a small reference current of nanoamperes.

\section{B. Temperature Dependence}

The temperature dependence of the mobility $\mu$ can be given by $\mu(T)=\mu\left(T_{0}\right)\left(T / T_{0}\right)^{-m}$, where $\mu\left(T_{0}\right)$ is the carrier 


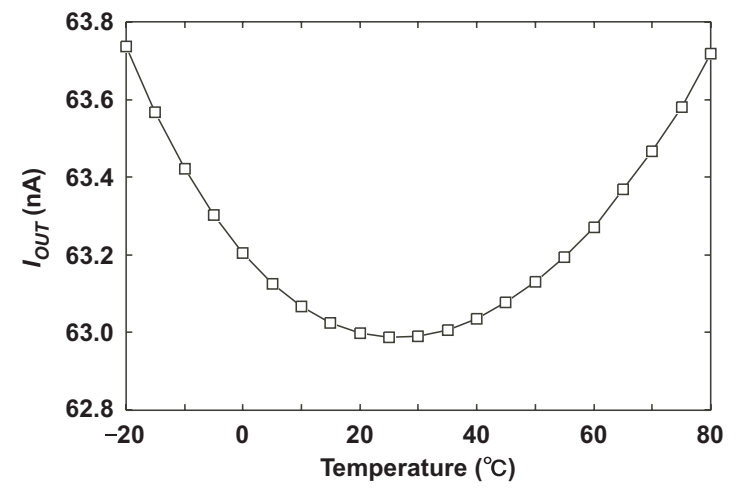

Fig. 2. Output current $I_{O U T}$ simulated as a function of temperature.

mobility at room temperature $T_{0}$, and $m$ is the mobility temperature exponent. The temperature coefficient (TC) of reference current $I_{O U T}$ in Eq. (7) is given by

$$
T C=\frac{1}{I_{\text {OUT }}} \frac{d I_{\text {OUT }}}{d T}=\frac{2-m-\frac{\Delta V_{T H}}{\eta V_{T}}}{T} .
$$

Therefore, the condition for a zero temperature coefficient can be given by

$$
2-m-\frac{\Delta V_{T H}}{\eta V_{T}}=0
$$

Setting $\Delta V_{T H}$ to an appropriate value can provide a zero TC at room temperature. The value of $V_{T H}$ can be adjusted by a bias voltage $V_{B}$. A sample circuit we designed showed a zero TC at $\Delta V_{T H}=47 \mathrm{mV}$.

\section{RESULTS}

We confirmed the operation of our reference circuit with the aid of SPICE simulation. We used the SPECTRE level 53 model with a parameter set for the $0.35-\mu \mathrm{m} 2 \mathrm{P} 4 \mathrm{M}$ standard CMOS process. The power supply voltage was set to $3 \mathrm{~V}$.

Figure 2 shows output current $I_{O U T}$ of the circuit as a function of temperature. The output current was about 63 $\mathrm{nA}$ and almost constant at temperatures -20 to $80{ }^{\circ} \mathrm{C}$. The temperature dependence and temperature coefficient were 7 $\mathrm{pA} /{ }^{\circ} \mathrm{C}$ and $115 \mathrm{ppm} /{ }^{\circ} \mathrm{C}$. Figure 3 shows a $\mathrm{TC}$ calculated from Eq. (8) and a TC simulated with SPICE, plotted as a function of temperature. The results obtained with Eq. (8) and SPICE simulation results correspond with each other.

To verify the stability of the circuit operation in device variation, we performed a corner analysis, using parameters provided by the manufacturer. The worst corners of nMOS and pMOS transistors (S: slow, T: typical, and F: fast) were taken into consideration. Figure 4 shows output current $I_{O U T}$ for five different process corner conditions. The value of $I_{O U T}$ changed with process corners. This is so because the difference in threshold voltages $\Delta V_{T H}$ depends on the process corners of nMOS devices. However, the reference current $I_{O U T}$ has little temperature dependence in each process corner condition.

Table I summarizes the performance of our reference circuits. The simulations showed the maximum power consumption of $1.9 \mu \mathrm{W}$ at $80{ }^{\circ} \mathrm{C}$. The line sensitivity was

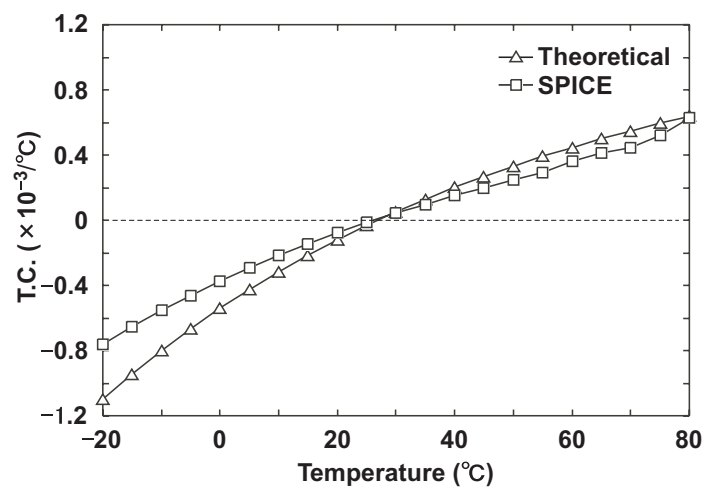

Fig. 3. TC of output current $I_{\text {OUT }}$ as a function of temperature; theoretical value obtained from Eq. (11) and SPICE simulated value.

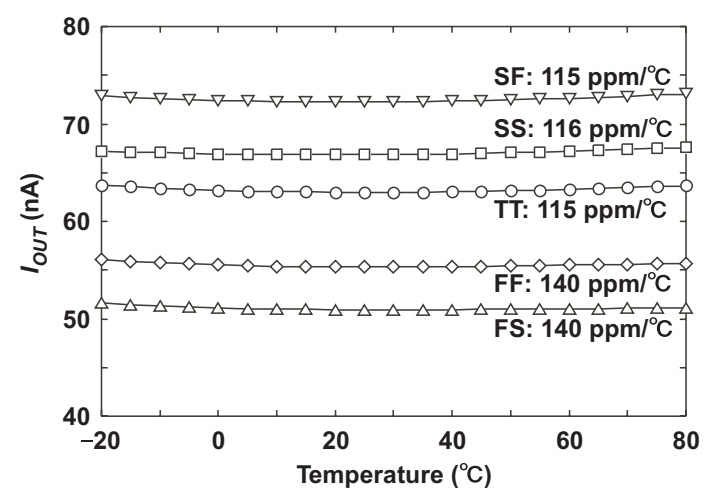

Fig. 4. Output current $I_{\text {OUT }}$ simulated with corner analysis. Process corners of nMOS and pMOS transistors were taken into consideration.

TABLE I. Performance summary

\begin{tabular}{c|c}
\hline Process & $0.35-\mu \mathrm{m}, 2-$ poly, 4-metal CMOS \\
Temperature range & $-20-80{ }^{\circ} \mathrm{C}$ \\
$V_{D D}$ & $2-3 \mathrm{~V}$ \\
$I_{O U T}$ & $63.3 \mathrm{nA}(\mathrm{Typical})$ \\
Power & $1.9 \mu \mathrm{W}\left(V_{D D}=3 \mathrm{~V}, T=80{ }^{\circ} \mathrm{C}\right)$ \\
$\mathrm{TC}$ & $115 \mathrm{ppm} /{ }^{\circ} \mathrm{C}$ \\
Line sensitivity & $0.08 \% / \mathrm{V}\left(V_{D D}=2-3 \mathrm{~V}\right)$ \\
\hline
\end{tabular}

$0.08 \% / \mathrm{V}$ in a $V_{D D}$ range of $2-3 \mathrm{~V}$. The lower limit of supply voltage can be reduced less than $2 \mathrm{~V}$ by using an operational amplifier instead of the cascode current mirrors we used in this example. Our circuit is useful as a reference current circuit for micro-power LSIs.

\section{REFERENCES}

[1] H. J. Oguey and D. Aebischer, "CMOS current reference without resistance," IEEE J. Solid-State Circuits, vol. 32, no. 7, pp. 1132-1135, July 1997.

[2] T. Hirose et al., "Ultralow-power current reference circuit with low temperature dependence," IEICE Trans. Electron., vol. E88-C, no. 6, pp. 1142-1147, June 2005.

[3] T. Hirose et al., "Temperature-compensated CMOS current reference circuit for ultralow-power subthreshold LSIs," IEICE Electronics Express, vol. 5, no. 6, pp. 204-210, March 2008.

[4] Y. Taur and T.H. Ning, Fundamentals of Modern VLSI Devices, Cambridge University Press, 2002. 